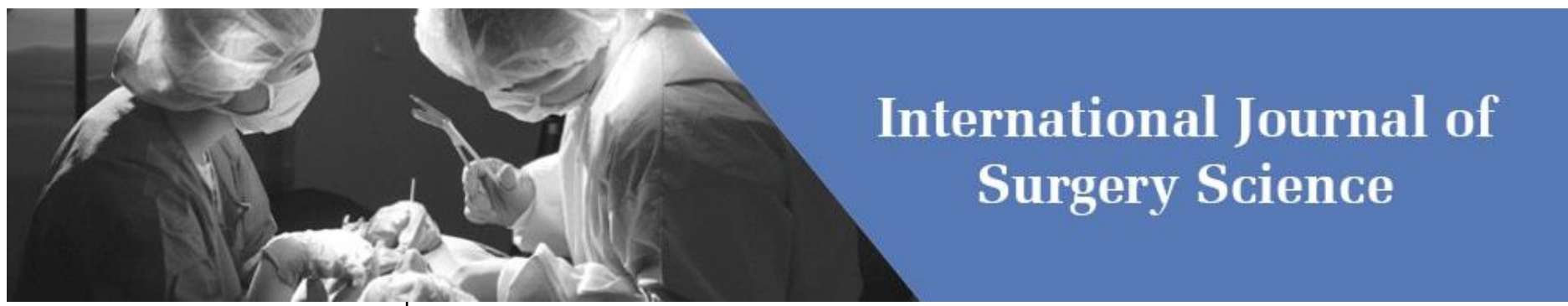

E-ISSN: 2616-3470

P-ISSN: 2616-3462

(C) Surgery Science

www.surgeryscience.com

2020; 4(1): 375-379

Received: 19-11-2019

Accepted: 21-12-2019

\section{Dr. Shivakumar S}

Associate Professor, Department of General Surgery, JSS Medical

College, JSS Academy of Higher

Education and Research, Mysore,

Karnataka, India

\section{Dr. Arjun MV}

Junior Resident, Department of General Surgery, JSS Medical

College, JSS Academy of Higher

Education and Research, Mysore,

Karnataka, India

\section{Dr. Diwakar SR}

Assistant Professor, Department of

General Surgery, JSS Medical

College, JSS Academy of Higher

Education and Research, Mysore,

Karnataka, India

\section{Dr. Sujith Kumar Sah}

Junior Resident, Department of General Surgery, JSS Medical

College, JSS Academy of Higher

Education and Research, Mysore,

Karnataka, India

\section{Dr. Madhava Shenoy}

Junior Resident, Department of General Surgery, JSS Medical College, JSS Academy of Higher Education and Research, Mysore, Karnataka, India

\section{Corresponding Author:} Dr. Arjun MV

Junior Resident, Department of General Surgery, JSS Medical College, JSS Academy of Higher Education and Research, Mysore, Karnataka, India

\section{Port vs 4 Port laparoscopic cholecystectomy in gall stones disease}

\author{
Dr. Shivakumar S, Dr. Arjun MV, Dr. Diwakar SR, Dr. Sujith Kumar Sah \\ and Dr. Madhava Shenoy
}

DOI: https://doi.org/10.33545/surgery.2020.v4.i1g.366

\section{Abstract}

Background: Gallstones or cholelithiasis constitute a significant health problem in developed societies and developing countries. We have shifted from open cholecystectomy to laparoscopic cholecystectomy as gold standard because of reduced morbidity and mortality. In this study, it is observed that just by reducing the port number, we could further reduce morbidity.

Methods of Study: Sixty patients who had gall stone disease and subjected to laparoscopic cholecystectomy were randomized to undergo either the 3 port or 4 port technique. Intraoperative Operative Time, Difficulty encountered, Post-operative pain was assessed on $12 \mathrm{hrs}, 24 \mathrm{hrs}$ pod3 and pod5, analgesic requirement after POD 1, Postoperative stay in hospital, return to normal activity

Results: Demographic data was comparable for both groups. Patient in the 3-port group had shorter operative time $(54.2 \pm 12.9$ min vs $75.8+26.28 \mathrm{~min})$, Reduced pain score, reduced analgesic usage(4.1vs 6.3 doses), early discharge( 3.6 vs 5 pod), early return to work (4.1 vs 5.8 )

Conclusion: Hence 3 port laparoscopic cholecystectomy is technically feasible, safe, and beneficial.

Keywords: Laparoscopic cholecystectomy

\section{Introduction}

Gallstones or cholelithiasis constitute a significant health problem in developed societies affecting $10 \%$ to $15 \%$ of the adult population, meaning 20 to 25 million Americans have gallstone. ${ }^{[1]}$ In India, the prevalence of gall stones or cholelithiasis is ranges from $10 \%$ to $20 \%$ in the adult population. It affects nearly $4.3 \%$ of the population ${ }^{[1]}$. Hence its significant health problem in India also. Gall stones or cholelithiasis is asymptomatic in majority of cases $(>80 \%)$. Approximately 1 to 2 percent of these patients develop symptoms and complication requiring surgery per year, 1 to 2 also constitute large numbers making cholecystectomy one of the most common operations performed by general surgeons ${ }^{[1]}$. Earlier Open cholecystectomy was the gold standard surgery for this gallstone diseases and later laparoscopic cholecystectomy was

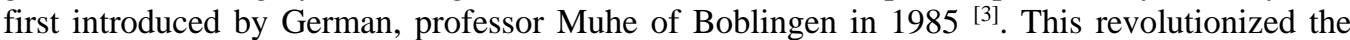
treatment of gallstone diseases and reduced postoperative morbidity significantly, complication rate, quicker postoperative recovery. And became the surgery of choice for the surgical removal of the gallbladder ${ }^{[3]}$.

For both chronic and acute calculus cholecystitis, which are the complication of cholelithiasis, standard treatment of choice is laparoscopic cholecystectomy with reduced postoperative morbidity, complication rate, quicker post-operative recovery ${ }^{[3]}$. But carries small increase in the rate of conversion. Hence they are also included in the study. So, the standard treatment of choice for high risk asymptomatic cholelithiasis, symptomatic cholelithiasis, chronic calculus cholelithiasis, acute calculus cholelithiasis is laparoscopic cholecystectomy. The standard laparoscopic cholecystectomy is done by using 4 ports. The fourth (lateral) port is used to grasp the fundus of the gallbladder so as to expose Calot's triangle. It is also seen that in experienced surgeon hand, many refinements in laparoscopic cholecystectomy has been tried which include reduction in port size. It has argued that laparoscopic cholecystectomy can be done safely without using the 4 port. By cooperative manipulation of the operative port instruments, Calot's triangle is exposed, dissected and gall bladder is dissected from gall bladder bed. Hence completing laparoscopic cholecystectomy in 3 ports only. Several studies have reported that 3 port laparoscopic cholecystectomy is technically possible ${ }^{[3,4,6,7,8]}$. 
In the era of laparoscopic surgery, early recovery and less postoperative pain are major goals to improve patient care and cut down cost. Several studies have demonstrated less postoperative pain and early recovery with reduction in size and number of ports. So we are conducting a randomised controlled study, to see the feasibility, safety, and benefits of 3-port laparoscopic cholecystectomy versus standard 4-port laparoscopic cholecystectomy ${ }^{[8]}$. Performance of laparoscopic cholecystectomy without much intraoperative difficulty of surgery in 3-port surgery was defined as technical feasibility. The surgery required conversion to 4 port because of technique was considered a technical failure. Performance of the procedure with comparable major complication like bleeding and injury to bile duct and any viscera with standard 4 port was defined as safety. Benefits in terms of reduced-(operative time, postoperative pain, postoperative analgesic usage, days of hospital stay days, days taken to return to work).

\section{Materials and Methods}

Description of study

a) Study Design: Randomised Comparative study,

b) Study place: Department of surgery, JSS hospital, JSS academy of higher education and Research, Mysore

c) Study duration: one and half year

d) Sample size:-sample size 60

e) Sampling technique and study population: Simple random selection: into 3 ports group and 4 ports group Nature of subject population: -all patients above 18year of age, sex, race included

f) Inclusion criteria: Acute calculus cholecystitis, Chronic calculus cholecystitis, Cholelithiasis

g) Exclusion criteria: Empyema of gall bladder, Mucocele of gall bladder, Acute cholecystitis with mass formation, Conversion from laparoscopic to open cholecystectomy

h) Study setting and Method of collection of data Data collection: Observation
Group selection: Simple Randomisation into port 3 group and port 4 group

Study Conduct stating the types of study activities that would be included in this section would be: medical history, USG abdomen and pelvis, Intraoperative details, Post-operative details

\section{Study Assessments of end points \\ Varaibles Studied \\ Intraoperatively \\ Operative time}

1. Intraoperative difficulty like calottes triangle dissection, cystic artery and duct ligation, dissection of gall bladder from liver bed

2. Rate of intraoperative complications

\section{Postoperatively}

1. Analgesic use post operatively after $24 \mathrm{hrs}$ of surgery

2. Post operative pain assessment using vas score

3. Hospital stay

4. Recovery to his daily work

\section{Methods of Study}

Sixty patients who had gall stone disease, who were eligible as per my inclusion criteria were included and all the patient were offered laparoscopic cholecystectomy, were randomized to undergo either the 3 port or 4 port technique. Four surgical tapes were applied to standard 4 port sites in both the group at end of the operation (single blinded). All dressing was kept intact for the 1 week after surgery. Data collected were intraoperative Operative Time, Rate of Intraoperative complication, Postoperative pain was assessed on 12hrs, $24 \mathrm{hrs}$, POD 3, POD 5 of surgery by using a $10 \mathrm{~cm}$ unscaled visual analogue scale (VAS), analgesic requirement after POD 1, Postoperative stay in hospital, return to normal activity.

Pain were assessed before giving analgesic. $10 \mathrm{~cm}$ unscaled visual analogue scale used shown

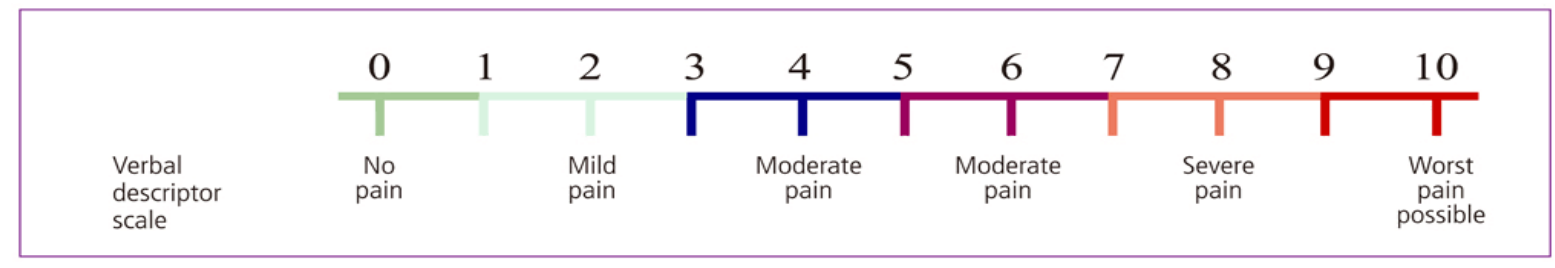

$10 \mathrm{~cm}$ unscaled visual analogue scale used shown

All patient received 2doses of analgesic on POD1 irrespective of pain score and for both the groups. Later analgesic dose was giving only if the pain score was above 4 and above. Pain score was assessed every 12 hourly and accordingly analgesic was given as described above. One dose of analgesic is equivalent to $50 \mathrm{mg}$ of diclofenac.

Patient was considered for discharge if his pain score was 2 and below, was free of other symptoms like vomiting, abdominal distension, fever, any other complication. If any above described complication was there patient discharge was deferred.

Return to normal activity was advised when the pain score was 1 or 0 and patient was free was all the symptom's.

\section{Statistical methods applied}

Descriptive Statistics: The Descriptives procedure displays univariate summary statistics for several variables in a single table and calculates standardized values (z scores). Variables can be ordered by the size of their means (in ascending or descending order), alphabetically, or by the order in which the researcher specifies. Following descriptive statistics were employed in the present study-, Mean, standard deviation, Frequency and percent

\section{Inferential Statistics}

Chi-Square Test: The Chi-Square Test procedure tabulates a variable into categories and computes a chi-square statistic. This goodness-of-fit test compares the observed and expected frequencies in each category to test either that all categories contain the same proportion of values or that each category contains a user-specified proportion of values.

Independent-Samples T Test: The Independent-Samples T Test procedure compares means for two groups of cases. Ideally, for this test, the subjects should be randomly assigned to two groups, so that any difference in response is due to the treatment (or lack of treatment) and not to other factors. 
Repeated Measure Anova: GLM Repeated Measures analyzes groups of related dependent variables that represent different measurements of the same attribute. This dialog box lets you define one or more within-subjects factors for use in GLM Repeated Measures. Note that the order in which you specify within-subjects factors is important. Each factor constitutes a level within the previous factor. All the statistical methods were carried out through the SPSS for Windows (version 16.0)

\section{Results}

Demographic data like age and sex in 3 port and 4 port group is are comparable. The significant finding on comparison of 3 port vs 4 port laparoscopic cholecystectomy was, patients who underwent 3 port surgery had shorter operative time than 4 port i.e. the mean operative time was $54.2667 \pm 12.921 \mathrm{mins}$ and $75.80 \pm 26.28$ respectively. And the mean difference of 21.5 with shorter operative time for 3 port laparoscopic cholecystectomy which is statistically highly significant. Operative time was calculated from the time of intubation to extubating.

Mean Pain score measured at $12 \mathrm{hrs}, 24 \mathrm{hrs}$, pod3 and pod5 was $4.4+1.6,3.6+1.5,2.33+2.3,0.433$ and $5+1.5,4.7+1.5,3.9+2.05$, $1.9+2.44$ in 3 port and 4 port group respectively. The statistical $\mathrm{P}$ value between 3 port and 4 port group was 0.15 at $12 \mathrm{hrs}$,
0.009 at 24hrs, 0.008 at POD3, 0.004 at POD 5 with VAS score lower at all intervals in 3 port group. Statistically significant VAS score was observed at 24hrs, POD3 and POD5 between 3 port and 4 port group with less VAS score for 3 port group. VAS score at $12 \mathrm{hrs}$ was not statistically significant, but was slightly less in 3 port group. When taken overall trend in the decrease of pain score was similar in 3 port and 4 port group but statistically 0.06 difference was between 3 port and 4port group with less VAS in 3 port group.

Mean POD of discharge was $3.66 \pm 1.29$ and $5.03 \pm 1.80$ in 3 port and 4 port groups respectively. POD at discharge was early in 3 port group compared with 4 port group which is statistically significant $(\mathrm{P}=0.01)$

Mean Analgesic use after $24 \mathrm{hrs}$ was $4.18 \pm 2.53$ doses and $6.38+4.00$ in 3 port and 4 port laparoscopic cholecystectomy respectively. Analgesic use was statistically significant $(\mathrm{P}=0.031)$ with higher analgesic requirement in 4 port group compared to 3 port group.

Means days of return to normal activity after surgery was $4.13 \pm 1,54$ and $5.80 \pm 2.31$ in 3 port and 4 port group respectively. Return to normal activity was again early in 3 port group compared to 4 Port group which was also statistically significant $(\mathrm{P}=0.02)$

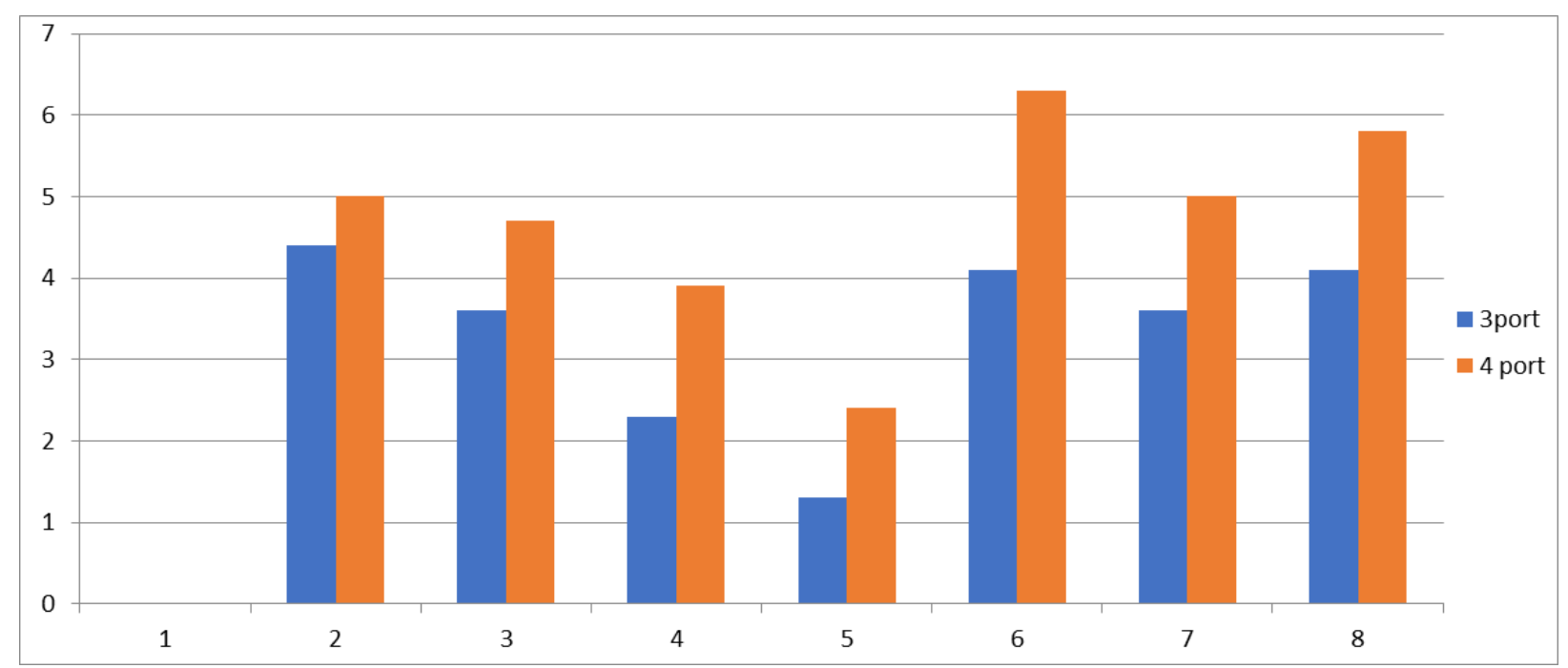

2 VAS12, 3 VAS24, 4- VASPOD3, 5 VASPOD5, 6 - No of Dosage of Analgesic use After 24 Hrs.,

7- POD at Discharge, 8 - Return to Normal Activity.

Graph 1: Comparison between 3 Port vs 4 Port Laparoscopic Cholecystectomy

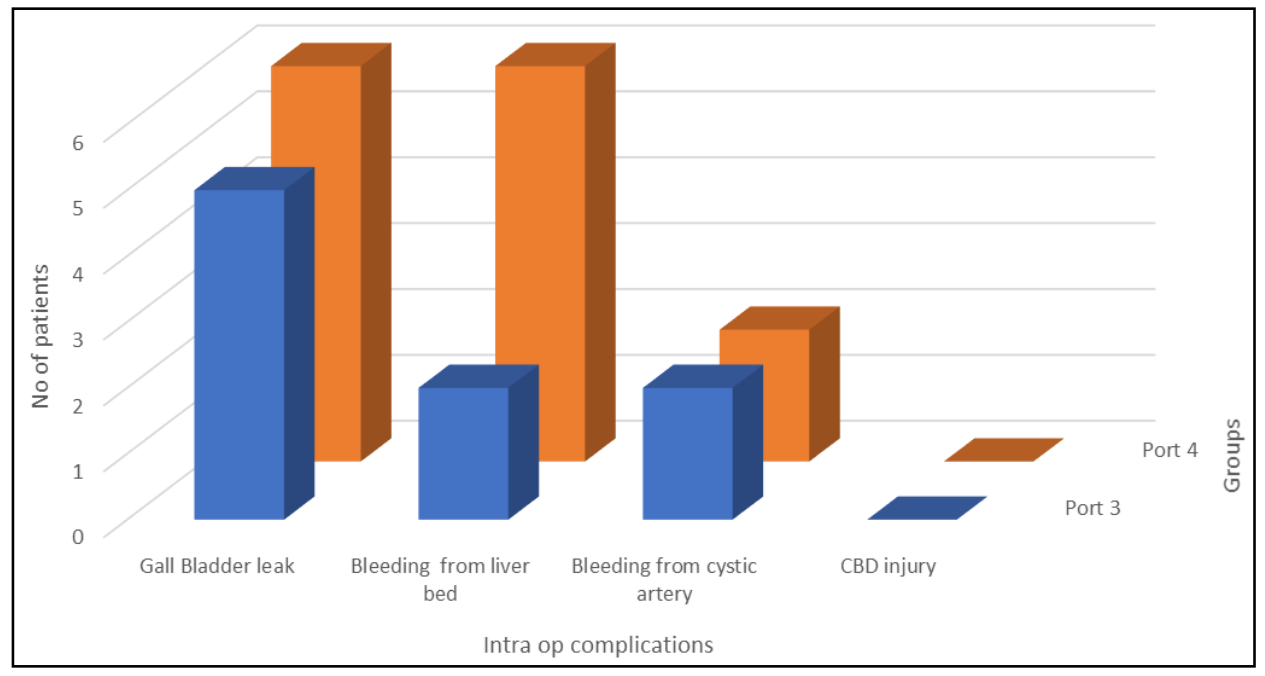

Graph 2: Intraoperative Complication of Laparoscopic Cholecystectomy 
Difficulty in dissection encountered occurred in 5 cases and 6 cases in 3port and 4 port group respectively out of 30 cases each. Hence Difficulty in dissection encountered incidence was similar between groups with no statistically significant difference

Similarly, cystic artery bleed, bleeding from liver bed and CBD injury was comparable between 3 port and 4 port groups. Cystic artery bleeding occurred in 4 cases i.e. is 2 each in 3 port and 4 port group. Bleeding from liver bed occurred in 8 cases with 2 in 3 port and 6 in 4 port group. CBD injury which is a dreaded complication of laparoscopic cholecystectomy did not occur in my study i.e. not a single case in both the groups.

4 cases which ever assigned for 3 port group were convert to 4 port because of intraoperative change in the diagnosis of the case.

\section{Discussion}

The main reason the laparoscopic surgeries become popular in 20th century is because of less post-operative pain, surgeries can be done as a day care surgery, early discharge, early recovery and early return to normal activity. Ultimately major goal of laparoscopic surgery was to achieve better patient care and cost effectiveness without increase complication, morbidity. It is observed in many studies that reduction in port number or size is associated with less post-operative pain, early recovery, early return to normal activity without increase in complication and morbidity.

So, in my study, I am comparing 3 port vs 4 port laparoscopic cholecystectomy. And in our study by reducing one port (retraction port) It was possible to reduce operative time with mean difference of 21.53 mins, reduced VAS score at $24 \mathrm{hrs}$, POD3, POD5 as shown in results, early discharge compared to 4 port group with mean difference of 1.36 days, decreased requirement of analgesics after $24 \mathrm{hrs}$ with mean difference of 2.20 doses, early return to normal activity with mean difference of 1.66 days which are all statistically significant as shown in results but there was no statistical significant change noted increase in intraoperative complication like Difficulty in dissection encountered, bleeding from liver bed, bleeding from cystic artery, CBD injury which is also shown in results.

\section{Operative Time}

One explanation for this reduction in operative time is less time spent on putting and closing the additional port ${ }^{[4]}$. One more important thing noticed in my study was, patient with longer operative time had higher visual analogue scale value, therefore, delaying the discharge and return to normal activity. Operative time was slightly higher in acute and chronic calculus cholecystitis cases because of difficulty in surgery and more incidence of intraoperative complication compared to simple cholelithiasis case

\section{Visual Analogue Scale Score}

Post-operative VAS for pain at $12 \mathrm{hrs}, 24 \mathrm{hrs}$, Pod 3 and Pod5 is less in 3 port group compared to 4 port group with $\mathrm{P}=1.53$ at $12 \mathrm{hrs}, 0.009$ at $24 \mathrm{hrs}, 0.008$ at Pod 3 and 0.004 at Pod 5 i.e. it was statistically significant at $24 \mathrm{hrs}$, Pod 3, pod 5. This increases in pain score was mainly because of one extra 4 port compared to 3 port i.e. that one extra site of pain ${ }^{[7]}$.

\section{POD at Discharge}

Patient in 3 port group have less pain compared to 4 port group hence we were able to discharge the patient early in 3 port group compared to 4 port group. But other factors considered for discharge like post-operative complication like vomiting, fever, etc. had no relation with the reduction in port number. But chance of post-operative complication like fever, vomiting, abdominal distension etc was slightly high in patients who had intraoperative complication.

\section{Return to Normal Activity}

Return to normal activity was also early in 3 port group compared to 4 port group i.e. with mean difference of 1.66 days. Its mainly attributed to reduced vas score. All procedure was done, by a single surgical specialist i.e. my guide so operator difference is nullified. No case was converted to 4 port because of intraoperative complications like bleeding, CBD injury etc. only 4 cases were converted to 4 port because of distended GB, enlarged left lobe of liver limited exposure of operative field. Similar success rate has been observed in other studies. One thing that was constantly observed in this series of 3 port laparoscopic cholecystectomy was difficulty in surgery with patient having long gall bladder, long peritoneal folds. It was mainly because fundus of the gall bladder, repeatedly fell forwards to the area of dissection of calot's triangle. Difficulty was also noted in patients with chronic cholecystitis, in dissecting Calot's because of chronic fibrosis. But overall, these difficulties were not significant, can be overcome by mastering 3 port surgery and also could be safely performed by experienced surgeon. Many surgeons have argued that since CBD cannot be visualised properly in 3 port laparoscopic cholecystectomy, chances of CBD duct injury is high. But In my study, there was no single case of CBD injury in both groups, it can be stated that in 3 port laparoscopic cholecystectomy CBD injury can prevent by gripping the infundibulum, retracting it laterally and dissecting at the infundibulum-cystic duct junction away from CBD. My study is comparable to the studies in the past i.e is there were no incidence of CBD injury, hence proving that chances of CBD injury with 3 port is similar to 4 port laparoscopic cholecystectomy in properly selected cases and in the hands of experienced surgeon who has mastered laparoscopic cholecystectomy in 3 port. Other intraoperative complication like bleeding from cystic artery, bleeding from liver bed. Difficulty in dissection encountered was statistically similar or comparable between the groups in the hands of experienced surgeon as proven in the results.

Interesting thing noted in my study was, when these intraoperative complications occurred invariably operative time was longer, so ultimately increasing vas score. It was also observed that patients who had these complications had increased chances of post-operative complication. As a result delaying discharge and return to normal activity. But these complications were independent of number of ports used in laparoscopic cholecystectomy.

\section{Conclusion}

At the end of my study, I conclude 3 port laparoscopic cholecystectomy is viable alternative to standard 4 port laparoscopic cholecystectomy in gall stone diseases like cholelithiasis, chronic calculus cholecystectomy, acute calculus cholecystitis in terms of safety, feasibility, and beneficial with reduced pain score.

\section{References}

1. Stinton LM, Shaffer EA. Epidemiology of gallbladder disease: Cholelithiasis and cancer. Gut and liver. 2012; 6(2):172.

2. Lundell L, Miettinen P, Myrvold HE, Hatlebakk JG, Wallen 
L, Maim A et al. Seven-year follow-up of a randomized clinical trial comparing proton-pump Inhibition with surgical therapy for reflux esophagitis. British Journal of Surgery. 2007; 94(2):198-203.

3. Al-Azawi D, Hussain N, Rayis AB, McMahon D, Hehir DJ. Three-port versus four-port laparoscopic cholecystectomy in acute and chronic cholecystitis. BMC surgery. 2007; 7(1):8.

4. Kumar M, Agrawal CS, Gupta RK. Three-port versus standard four-port laparoscopic cholecystectomy: a randomized controlled clinical trial in a community-based teaching hospital in eastern Nepal. JSLS: Journal of the Society of Laparoendoscopic Surgeons. 2007; 11(3):358.

5. Leggett PL, Bissell CD, Churchman-Winn R, Ahn C. Three-port microlaparoscopic cholecystectomy in 159 patients. Surgical endoscopy. 2001; 15(3):293-6.

6. Trichak S. Three-port vs standard four-port laparoscopic cholecystectomy. Surgical endoscopy. 2003; 17(9):1434-6.

7. Cerci C, Tarhan OR, Barut I, Bülbül M. Three-port versus four-port laparoscopic cholecystectomy. Hepatogastroenterology. 2007; 54(73):15-6.

8. Mujahid MD, Hameed F, Riaz O, Saleem M, Hussain R. Three Port Versus Four Port Laparoscopic Cholecystectomy. Group. 2011; 20:25.

9. Miettinen P, Myrvold HE, Rattleback JG, Wallin L, Malm A, Sutherland I et al. Seven-year follow-up of a randomized clinical trial comparing proton-pump inhibition with surgical therapy for reflux esophagitis. British Journal of Surgery. 2007; 94(2):198-203 